\title{
Las universidades españolas en Twitter: Mensajes, contenidos y públicos
}

\author{
Bernardo J. GómEZ CALDERóN \\ Universidad de Málaga \\ bjgomez@uma.es \\ Francisco Javier Paniagua Rojano \\ Universidad de Málaga \\ fjpaniagua@uma.es
}

\section{Resumen:}

En apenas un lustro, el uso de Twitter se ha generalizado entre las universidades españolas. El 97,4 por ciento de ellas dispone de perfil institucional en esta red social, caracterizada por la brevedad de sus mensajes y la posibilidad de acceder a la información sin necesidad de ser usuario. El empleo de Twitter por parte de las universidades presenta características bien definidas, como pone de manifiesto este trabajo: público preferentemente estudiantil, contenidos relacionados sobre todo con la docencia y la extensión universitaria, y poca atención a la actividad investigadora.

Palabras clave: Universidad; Comunicación Institucional; redes sociales; Twitter.

\section{The Spanish universities in Twitter: Messages, contents and publics}

\begin{abstract}
:
The use of Twitter has become widespread among the Spanish universities since five years ago. The 97.4 per cent of them have got an institutional profile in this social network, whose most prominent properties are the brevity of their messages and the easiness to access to the information for all the users. This study demonstrates that the use of Twitter by the Spanish universities shows some clear characteristics: messages orientated to the students, contents connected with educational and cultural activities and lack of information about university research.
\end{abstract}

Key Words: University; Institutional Communication; social networks; Twitter.

\section{Referencia normalizada:}

Gómez Calderón, B.J. y Paniagua Rojano, F.J. (2014): Las universidades españolas en Twitter: Mensajes, contenidos y públicos. Historia y Comunicación Social. Vol. 19. Núm. Especial Enero. Págs. 681-694.

Sumario: 1. Introducción. 1.1. Aplicaciones de Twitter en el entorno universitario. 1.2. La implantación de Twitter en el sistema universitario español. 2. Objeto. 3. Metodología. 4. Resultados. 4.1. Origen del tweet. 4.2. Ámbito del tweet. 4.3. Temas abordados. 4.4. Enlaces contenidos en los tweets. 4.5. Público al que se dirigen los tweets. 5. Conclusiones. 6. Referencias bibliográficas. 7. Notas. 


\section{Introducción}

En los últimos cinco años, las universidades españolas se han incorporado de forma generalizada a las redes sociales, transformando de raíz sus hábitos comunicativos. Si en 2010 apenas una decena de ellas contaban con perfiles en alguna social network (Aguilera, Farias y Baraybar, 2010), hoy no hay prácticamente ninguna que no disponga de varias cuentas oficiales con miles de seguidores.

La utilidad más directa que las universidades encuentran en las redes es la de comunicarse con sus públicos mayoritarios, los alumnos, de forma rápida y eficaz (Gómez, Roses y Farias, 2012). No puede obviarse que, para los estudiantes, el empleo de aplicaciones 2.0 es un componente esencial de su alfabetización mediática; su conocimiento y dominio por parte de las instituciones de educación superior resulta, por lo tanto, crucial (Tapia et al., 2010).

Son muchas las utilidades que las universidades pueden extraer de las redes, más allá de mantener el contacto diario con sus alumnos; también es una herramienta para continuar vinculadas a quienes han terminado ya sus estudios, para darse a conocer entre públicos externos, para mejorar su imagen corporativa (Kierkegaard, 2010) y, dada la extensión de este tipo de herramientas entre los adolescentes, para acercarse a quienes aún no han completado la enseñanza obligatoria (Regalado, 2011).

En el ámbito de las redes, una moda sucede a otra, y propuestas que son en un momento dado las más populares pierden fuelle con rapidez, en parte debido a la velocidad con que se desarrollan nuevas soluciones telemáticas. De ahí que Twitter se esté imponiendo en ciertos ámbitos a Facebook y se haya convertido en la red preferida por muchos departamentos de comunicación universitarios a la hora de mantener informados a sus públicos.

\subsection{Aplicaciones de Twitter en el entorno universitario}

Lo que tienen en común todas las redes sociales ${ }^{1}$ es que permiten formar comunidades virtuales de usuarios que interactúan a través de ellas de modo instantáneo (Ebner et al., 2010). La orientación de los contenidos de cada red varía: hay redes temáticas (como Nvivo, dedicada a la música) y las hay de carácter profesional (Linkedin), pero las más comunes son las de tipo generalista (Celaya, 2008), es decir, networks que admiten cualquier tipo de contenido y que tienen un alcance, en cuanto a número de usuarios, mucho más amplio. En esta categoría entran las más extendidas: MySpace, Facebook, Tuenti y, por supuesto, Twitter.

Twitter, un servicio de microblogging creado en los Estados Unidos en 2006, ha crecido exponencialmente en el último lustro: hoy cuenta con 232 millones de usuarios $^{2}$ (lejos, en cualquier caso, de los 1.155 millones de Facebook ${ }^{3}$, pero muy por delante del resto de competidoras). El modo en que se generan y comparten los contenidos en esta red explica su rápida aceptación: los mensajes no admiten más de 140 caracteres (Veletsianos, 2012); existe la posibilidad de entablar conversaciones sobre temas o eventos concretos a través del empleo de hashtags; y se puede acceder 
a la información emitida por cualquier usuario sin necesidad de disponer de cuenta propia (Arana et al., 2012). Por otro lado, su carácter multimedia y la posibilidad de documentar las conversaciones (Guzmán et al., 2012) son también ventajas claras frente a otras social networks.

En el ámbito universitario, Twitter se perfila actualmente como una herramienta de doble aplicación: por un lado, sirve de apoyo a la docencia, cuando la red se inserta en el proceso de enseñanza-aprendizaje y se dota a sus contenidos de finalidad didáctica. La dinámica educativa clásica se ve así alterada, con resultados provechosos según todos los análisis (cf. Altamirano et al., 2009; Guzmán et al., 2012). Los estudiantes, en general, responden positivamente a la utilización de Twitter en el aula, incrementándose su interés por las materias explicadas (Rinaldo et al., 2011). Se trata, no obstante, de un empleo de la red que aún no está consolidado, y en muchos casos se echa en falta una planificación más rigurosa de las actividades por parte del personal docente (Roses, Gómez y Farias, 2012).

Pero además, Twitter es una herramienta cada vez más importante en las tareas de difusión de información corporativa, que es la vertiente que interesa aquí. A través de sus perfiles oficiales, las universidades dan a conocer desde actividades docentes extracurriculares -del tipo congresos o cursos- hasta noticias relacionadas con la actualidad de la institución, pasando por eventos culturales o, directamente, campañas promocionales destinadas a la captación de alumnos o a la fidelización de los públicos internos. Los gabinetes de comunicación universitarios encuentran en Twitter un escaparate fácilmente accesible, en el que los mensajes se consumen de forma inmediata y se replican sin coste alguno para la organización; por este motivo, su incorporación a la red ha sido masiva.

\subsection{La implantación de Twitter en el sistema universitario español}

En la actualidad, el 97,4 por ciento de las universidades españolas dispone de cuenta oficial en Twitter ${ }^{4}$. La progresión de esta cifra ha sido exponencial, dado que la primera que se incorporó a la red, la Oberta de Catalunya, lo hizo el 27 de mayo de 2008. Antes de que finalizara el año, se sumaron otras nueve; en 2009 lo hicieron 19; en 2010, 22; en 2011, 18; en 2012 se unieron cinco más, y en 2013, una.

Si se analiza el contenido y el grado de actividad que cada universidad exhibe en Twitter, la conclusión es que su empleo presenta características heterogéneas, y si bien se aprecian tendencias mayoritarias, no hay, desde luego, patrones fijos. Como constatación general, puede afirmarse que no existe correlación entre número de estudiantes y número de seguidores, ni entre ambos y la cantidad de mensajes emitidos por cada institución (Tabla 1). Así, se localizan universidades con pocos alumnos pero muy activas y otras con un volumen de estudiantes elevado pero pocos followers -en proporción pero también en términos absolutos- en su cuenta oficial ${ }^{5}$. Desde el punto de vista cuantitativo, por tanto, el comportamiento es diverso (Paniagua, Gómez y Fernández, 2012). 


\begin{tabular}{|l|c|c|}
\hline \multicolumn{2}{|c|}{ Tabla 1. Universidades españolas con mayor número de alumnos } \\
\hline & $\begin{array}{c}\mathrm{N}^{0} \text { alumnos } \\
2012-13\end{array}$ & $\begin{array}{c}\text { Seguidores en } \\
\text { Twitter* }\end{array}$ \\
\hline $\begin{array}{l}\text { Nacional de Educación a Distancia } \\
\text { (UNED) }\end{array}$ & 260.079 & 58.499 \\
\hline Barcelona & 87.486 & $20.976^{* *}$ \\
\hline Complutense de Madrid & 85.236 & 24.206 \\
\hline Sevilla & 58.217 & 47.338 \\
\hline Granada & 56.820 & 57.110 \\
\hline València & 47.063 & 17.103 \\
\hline País Vasco & 45.000 & 9.784 \\
\hline Oberta de Catalunya & 38.842 & 18.574 \\
\hline Autònoma de Barcelona & 37.660 & 12.482 \\
\hline Málaga & 35.709 & 33.078 \\
\hline
\end{tabular}

*Cifras a 25 de octubre de 2013

** Total agregado de los tres perfiles generalistas (en catalán, castellano e inglés) Fuentes: universidades, Twitter. Elaboración propia.

No abundan los análisis sobre el tipo de mensajes que las universidades difunden a través de Twitter -lo que podríamos considerar la dimensión cualitativa de su utilización-, pero los aparecidos hasta la fecha apuntan a un predominio claro de las noticias relacionadas con la actualidad institucional, la docencia y las actividades formativas extracurriculares (Reina, Fernández y Noguer, 2012). Por su parte, Guzmán et al. (2012: 34), en un estudio basado en la utilización de hashtags cuya muestra incluye a las nueve universidades españolas mejor situadas en el ranquin de Shanghái, obtienen una distribución de contenidos que matiza y completa las conclusiones anteriores y evidencia la doble finalidad - didáctica y corporativa- que Twitter tiene en este segmento ${ }^{6}$.

\section{Objeto}

La incorporación a Twitter se ha generalizado entre las universidades españolas. Ahora bien, ¿qué uso se le da a esta potente herramienta de comunicación? El presente trabajo surge del interés por determinar las características de la información que las instituciones de educación superior difunden a través de sus perfiles oficiales, con el objetivo de disponer de un molde descriptivo satisfactorio pero también de detectar eventuales deficiencias que puedan incidir negativamente en la imagen de marca-el ethos institucional- que se transmite.

De este modo, se definieron los siguientes objetivos de investigación: 
- Establecer las características de la actividad en Twitter de las universidades españolas, tomando como referencia a aquellas instituciones con mayor tráfico de contenidos en esta red social.

- Determinar las propiedades de la información que las universidades vehiculan a través de sus cuentas institucionales en Twitter, y a qué tipo de elementos aparece asociada.

- Perfilar a qué público o públicos se dirigen los mensajes emitidos.

\section{Metodología}

Para alcanzar los objetivos propuestos, se decidió aplicar como método el análisis de contenido, escogido por su idoneidad para afrontar investigaciones cuantitativas sobre textos escritos. A través de él, se pueden recopilar, procesar y evaluar grandes cantidades de información (Sánchez, 2005: 214). Esto, unido a su frecuente utilización en la descripción de los componentes de los mensajes mediáticos (Igartua, 2006: 194), lo convertían en la técnica más apropiada para este estudio.

El universo sobre el que se trabajó fue el de las 75 universidades españolas que poseen cuenta oficial en Twitter ${ }^{7}$. Para determinar la muestra, se optó por seleccionar a aquellas que exhibieran mayor nivel de actividad en la red, definiendo dicho nivel en función del promedio de mensajes emitidos por cada perfil institucional desde su fecha de creación ${ }^{8}$. De la clasificación obtenida se seleccionaron las cuentas con un índice superior a ocho (Tabla 2):

\begin{tabular}{|c|c|c|c|c|c|c|c|c|}
\hline & & $\begin{array}{c}\mathrm{N}^{0} \text { de } \\
\text { alumnos }\end{array}$ & Seguidores & Siguiendo & $\begin{array}{l}\mathrm{N}^{o} \text { de } \\
\text { tweets }\end{array}$ & Cuenta & \begin{tabular}{|c} 
Fecha de \\
creación de la \\
cuenta
\end{tabular} & $\begin{array}{c}\text { Promedio } \\
\text { de tweets } \\
\text { al dia }\end{array}$ \\
\hline 1 & Murcia & 27.440 & 20.649 & 14.984 & 21.963 & (a) umnoticias & 26-VII-2010 & 20,26 \\
\hline 2 & Miguel de Cervantes & 1.514 & 2.249 & 992 & 12.712 & (a)UEMC & 28-II-2011 & 14,66 \\
\hline 3 & Burgos & 8.286 & 4.441 & 202 & 8.777 & (a) UBUEstudiantes & 17-III-2011 & 10,33 \\
\hline 4 & Granada & 62.706 & 49.459 & 49.145 & 10.066 & (a) canalUGR & $17-\mathrm{V}-2010$ & 8,72 \\
\hline 5 & Málaga & 34.891 & 27.880 & 2.912 & 9.940 & @infouma & 9-III-2010 & 8,13 \\
\hline
\end{tabular}

*Cifras a 13 de julio de 2013. Fuente: páginas webs de universidades, Twitter, http://tweetgrader.com/go/tweetingsince. Elaboración propia.

Posteriormente, se recuperaron todos los tweets generados por cada una de las cinco universidades escogidas durante una "semana tipo" construida entre los meses de abril y junio de 2013, comenzando de forma aleatoria por el sábado 27 de abril. El total de mensajes seleccionados ascendió a 597 (Tabla 3). 


\begin{tabular}{|l|c|c|c|c|c|c|c|c|}
\hline & & & & & & & \\
& S 27-abr & D 5-may & L 6-may & M 14-may & X 22-may & J 30-may & V 7-jun & Total \\
\hline Murcia & 30 & 17 & 40 & 38 & 55 & 40 & 48 & 268 \\
\hline $\begin{array}{l}\text { Miguel de } \\
\text { Cervantes }\end{array}$ & 0 & 1 & 18 & 9 & 7 & 19 & 21 & 75 \\
\hline Burgos & 8 & 8 & 26 & 32 & 13 & 11 & 6 & 104 \\
\hline Granada & 4 & 8 & 20 & 10 & 12 & 18 & 14 & 86 \\
\hline Málaga & 0 & 0 & 3 & 8 & 22 & 24 & 5 & 62 \\
\hline Total & 42 & 34 & 107 & 97 & 111 & 112 & 94 & 597 \\
\hline
\end{tabular}

Fuente: Twitter. Elaboración propia.

La ficha de análisis aplicada a cada tweet contemplaba los siguientes ítems: a) origen (la propia universidad, otros usuarios -en el caso de mensajes de respuesta-o híbrido - retweets-); b) ámbito temático (según el mensaje abordara la actualidad de la universidad, la de otras instituciones, la de otras universidades o la del sistema educativo en general); c) asunto (estructurado en seis categorías: actos institucionales, cuestiones administrativas, actividad académica, actividad investigadora, extensión universitaria y otros); d) público del tweet (estudiantes, PDI, PAS, comunidad universitaria, colectivos externos o destinatario universal); e) enlace incorporado (que podía ser a nota elaborada por el gabinete de prensa de la institución; a información aparecida en medios; a página web, cuenta de Twitter o Facebook de la universidad; a página o cuenta de Twitter externa; a la sala de prensa virtual o a la agenda de eventos); f) tipo de retweet (de estudiantes, de PDI, de PAS, de otras cuentas de la universidad o de usuarios externos, y sobre qué temas); y g) tipo de mensajes de respuesta emitidos (de solución de consultas, de disculpas o de agradecimiento).

\section{Resultados}

\subsection{Origen del Tweet}

Lo más habitual es que sean las universidades las que generen la mayor parte de los mensajes difundidos en sus cuentas oficiales. Así, del total de tweets seleccionados, un 64,3 por ciento tiene su origen en las propias instituciones. No obstante, hay variaciones en esta tendencia, de ahí que las proporciones oscilen entre el 34,7 por ciento de la Universidad Miguel de Cervantes y el 79,8 de la de Burgos (Tabla 4).

Menos numerosas son las respuestas a usuarios (16,2 por ciento de la muestra), dirigidas por lo general a estudiantes, aunque también hay aquí divergencias entre unos perfiles y otros, siendo los índices más alejados del promedio los de Burgos (1 por ciento) y Granada (43 por ciento). 
En cuanto a los retweets, presentan una tasa media similar a la anterior $(19,4$ por ciento), registrándose de nuevo algunas cifras llamativas, bien por exiguas (7 por ciento en el caso de la UMA), bien por generosas (37,3 en el de la UEMC).

\begin{tabular}{|l|c|c|c|c|c|c|}
\hline \multicolumn{7}{|c|}{ Tabla 4. Origen del tweet } \\
\hline & UMU & UEMC & UBU & UGR & UMA & Total \\
\hline Universidad & 73,0 & 34,7 & 79,8 & 50,0 & 56,5 & 64,3 \\
\hline Usuario & 11,1 & 28,0 & 1,0 & 43,0 & 12,9 & 16,2 \\
\hline Retweet & 15,9 & 37,3 & 19,2 & 7,0 & 30,6 & 19,4 \\
\hline Total & 100,0 & 100,0 & 100,0 & 100,0 & 100,0 & 100,0 \\
\hline
\end{tabular}

* Cifras expresadas en tantos por ciento.

El origen de la mayoría de los retweets se localiza en cuentas externas $(49,1$ por ciento del total). Lo más frecuente es que las universidades repliquen comentarios de otras instituciones educativas, de organismos responsables de actividades culturales o de medios de comunicación que informan sobre actividades en el campus (Tabla 5). Los mensajes de los alumnos representan el 27,6 por ciento de los casos identificados, gracias sobre todo a las aportaciones de la UBU y la UEMC, cuyas altas tasas -superiores a los cuarenta puntos- contrastan con el rotundo 0 por ciento cosechado por la Universidad de Málaga.

\section{2. Ámbito del Tweet}

La actualidad de cada universidad acapara, como es natural, la mayor parte de la información que se suministra a través de las cuentas oficiales de Twitter. No obstante, tratándose de un canal tan dinámico, con muchos miles de seguidores altamente cualificados, llama la atención que apenas se preste atención a otros ámbitos de la actualidad o el entorno que, por lógica, deberían despertar el interés de profesores y alumnos.

Así, las menciones que reciben otras instituciones de educación superior es muy reducida (1,2 por ciento), e incluso el sistema universitario obtiene una tasa apenas testimonial $(2,2)$, y ello en un momento en el que su mantenimiento y eventual reforma ocupa un lugar destacado en la agenda de los medios de comunicación (Tabla 5). En este sentido, la focalización referencial de los contenidos de Twitter es muy elevada.

\begin{tabular}{|l|c|c|c|c|c|c|}
\hline \multicolumn{7}{|c|}{ Tabla 5. Ámbito del tweet } \\
\hline & UMU & UEMC & UBU & UGR & UMA & Total \\
\hline Universidad & 81,5 & 97,3 & 91,3 & 96,5 & 91,9 & 88,4 \\
\hline Otras instituciones & 12,6 & 2,7 & 5,8 & 2,3 & 8,1 & 8,2 \\
\hline Otras universidades & 1,9 & 0,0 & 1,0 & 1,2 & 0,0 & 1,2 \\
\hline Sistema educativo & 4,1 & 0,0 & 1,9 & 0,0 & 0,0 & 2,2 \\
\hline Total & 100,0 & 100,0 & 100,0 & 100,0 & 100,0 & 100,0 \\
\hline
\end{tabular}




\subsection{Temas abordados}

No todas las parcelas de la actividad universitaria generan el mismo volumen de contenidos en la red: hay esferas muy visibles y otras prácticamente inexistentes. De hecho, el 96,3 por ciento de los tweets analizados giran en torno a sólo cinco temas (Tabla 6):

\begin{tabular}{|l|c|c|c|c|c|c|}
\hline \multicolumn{7}{|c|}{ Tabla 6. Temas abordados } \\
\hline & UMU & UEMC & UBU & UGR & UMA & Total \\
\hline Temas institucionales & 12,2 & 2,7 & 4,8 & 0,0 & 12,9 & 8,0 \\
\hline Temas administrativos & 4,8 & 1,3 & 13,5 & 39,5 & 17,7 & 12,2 \\
\hline Docencia & 45,6 & 50,7 & 43,3 & 26,7 & 38,7 & 42,4 \\
\hline Actividad investigadora & 11,9 & 9,3 & 6,7 & 10,5 & 9,7 & 10,2 \\
\hline Extensión universitaria & 22,2 & 30,7 & 27,9 & 20,9 & 16,1 & 23,5 \\
\hline Otros temas & 3,3 & 5,3 & 3,8 & 2,3 & 4,8 & 3,7 \\
\hline Total & 100,0 & 100,0 & 100,0 & 100,0 & 100,0 & 100,0 \\
\hline
\end{tabular}

a) Docencia: la actividad académica representa el principal foco de interés de las cuentas universitarias de Twitter (42,4 por ciento de incidencias) (Tabla 7). Por lo general, se emplean para el anuncio de cursos, congresos, talleres o conferencias (72,4 por ciento), que son iniciativas naturalmente necesitadas de difusión. En otros casos, los mensajes hacen referencia a novedades que tienen que ver con títulos de grado o posgrado impartidos por la universidad (16,9 por ciento).

\begin{tabular}{|l|c|c|c|c|c|c|}
\hline \multicolumn{7}{|c|}{ Tabla 7. Tema: Docencia } \\
\hline & UMU & UEMC & UBU & UGR & UMA & Total \\
\hline $\begin{array}{l}\text { Cursos, congresos, } \\
\text { talleres, conferencias }\end{array}$ & 78,0 & 62,5 & 64,7 & 57,1 & 85,0 & 72,4 \\
\hline $\begin{array}{l}\text { Declaraciones de } \\
\text { profesores (expertos) }\end{array}$ & 1,7 & 0,0 & 0,0 & 0,0 & 0,0 & 0,9 \\
\hline Docencia reglada & 11,9 & 37,5 & 20,6 & 19,0 & 5,0 & 16,9 \\
\hline Becas & 5,1 & 0,0 & 14,7 & 19,0 & 10,0 & 7,6 \\
\hline Otros & 3,4 & 0,0 & 0,0 & 4,8 & 0,0 & 2,2 \\
\hline Total & 100,0 & 100,0 & 100,0 & 100,0 & 100,0 & 100,0 \\
\hline
\end{tabular}

b) Extensión universitaria: segundo ámbito temático en términos cuantitativos, incluye todas las actividades relacionadas con la cultura, el deporte, la asistencia a la comunidad o la responsabilidad social corporativa impulsadas desde los campus. Su promedio es notable, 23,5 por ciento, y no hay universidades que destaquen frente a otras en esta parcela, que concita un interés uniforme. Dentro de las distintas categorías, la información sobre eventos culturales es la más frecuente. 
c) Temas administrativos: abarca asuntos como la difusión de convocatorias públicas, la aprobación de normativas o la información sobre plazos y procedimientos. En este caso, la tasa de incidencia es del 12,2 por ciento, si bien hay universidades, como la de Granada, que elevan la proporción hasta casi el 40.

d) Investigación: comprende un repertorio amplio de actividades, que van desde la divulgación de trabajos realizados por el personal docente hasta la concesión de premios y distinciones, pasando por el anuncio de publicaciones y tesis doctorales, la convocatoria de ayudas y la suscripción de convenios específicos.

La dimensión investigadora de las universidades es indisociable de la educativa, al menos en el caso español. Ambas facetas contribuyen a definir el alcance y la calidad del trabajo que se realiza en los campus. Resulta chocante, por tanto, que las referencias en Twitter a las novedades que tienen que ver con la investigación sean tan escasas: sólo el 10,2 por ciento de los mensajes seleccionados se ocupa de ellas.

e) Actualidad institucional: finalmente, los perfiles oficiales de Twitter reservan una cuota de mensajes para dar cuenta de los asuntos relacionados con el gobierno y la gestión de las universidades ( 8 por ciento de tweets analizados). En ellos se informa, principalmente, de inauguraciones o clausuras, reuniones de órganos directivos, firmas de convenios, entregas de premios, actos de graduación, concesión de doctorados honoris causa y celebraciones y homenajes a miembros de la comunidad.

\subsection{Enlaces contenidos en los Tweets}

Como el resto de redes sociales, Twitter permite incorporar enlaces a documentos externos, algo que si en otros casos resulta enriquecedor, aquí se vuelve casi imprescindible dada la brevedad de los mensajes. La muestra revela una notable variedad de elementos asociados a los tweets: la mayor parte de ellos ofrecen enlaces a la web de la propia institución (25,2 por ciento de casos) o a noticias aparecidas en prensa que abordan la actualidad del campus (24,8 por ciento).

Menos frecuentes son los links a materiales elaborados por el personal de comunicación de las universidades, sólo un 9,9 por ciento, si bien las cuentas de la UEMC y la UBU los incluyen de modo más habitual. Aquellos gabinetes que poseen salas de prensa virtuales, los de Murcia y Málaga, enlazan con ellas regularmente, y lo mismo hacen ambos con los perfiles que sus universidades mantienen en otras redes sociales, como Facebook (Tabla 8). 


\begin{tabular}{|l|c|c|c|c|c|c|}
\hline \multicolumn{7}{|c|}{ Tabla 8 . Enlace a.. } \\
\hline & UMU & UEMC & UBU & UGR & UMA & Total \\
\hline Nota propia & 3,4 & 20,5 & 25,6 & 9,3 & 0,0 & 9,9 \\
\hline Noticia en medios & 34,3 & 12,8 & 28,9 & 0,0 & 13,0 & 24,8 \\
\hline Web de la Universidad & 2,9 & 15,4 & 38,9 & 83,3 & 39,1 & 25,2 \\
\hline Web o Twitter externos & 6,3 & 51,3 & 6,7 & 7,4 & 6,5 & 10,6 \\
\hline Sala de prensa & 14,5 & 0,0 & 0,0 & 0,0 & 17,4 & 8,7 \\
\hline Agenda & 36,7 & 0,0 & 0,0 & 0,0 & 2,2 & 17,7 \\
\hline $\begin{array}{l}\text { Otro Twitter de la } \\
\text { Universidad }\end{array}$ & 0,5 & 0,0 & 0,0 & 0,0 & 4,3 & 0,7 \\
\hline $\begin{array}{l}\text { Facebook de la } \\
\text { Universidad }\end{array}$ & 1,4 & 0,0 & 0,0 & 0,0 & 17,4 & 2,5 \\
\hline Total & 100,0 & 100,0 & 100,0 & 100,0 & 100,0 & 100,0 \\
\hline
\end{tabular}

* Cifras expresadas en tantos por ciento.

\subsection{Público al que se dirigen los Tweets}

Por definición, Twitter es una red generalista. A ella accede todo tipo de usuarios, y en las cuentas oficiales universitarias figura un repertorio de seguidores muy diverso. No obstante, en la práctica, es la propia comunidad educativa la más interesada en estos perfiles, con especial incidencia entre los estudiantes, que son los que mejor conocen las aplicaciones 2.0.

Su potencial de cara a los públicos externos, sin embargo, es elevado; no en vano, las instituciones emplean habitualmente Twitter como plataforma desde la que labrarse una imagen de marca atractiva. Por ello, en torno al 50 por ciento de la muestra lo componen mensajes de destinatario universal o dirigidos a usuarios no pertenecientes al colectivo universitario (Tabla 9).

Por otro lado, la mayor parte de los tweets de consumo interno tienen en los alumnos a su target group (36,5 por ciento), mientras que el PDI y el PAS apenas parecen contar para los gestores de las cuentas analizadas: su tasa de incidencia va del 0,2 al 0 por ciento. 


\begin{tabular}{|l|c|c|c|c|c|c|}
\hline \multicolumn{7}{|c|}{ Tabla 9. Público de los tweets } \\
\hline & UMU & UEMC & UBU & UGR & UMA & Total \\
\hline Estudiantes & 12,6 & 41,3 & 47,1 & 83,7 & 51,6 & 36,5 \\
\hline PDI & 0,4 & 0,0 & 0,0 & 0,0 & 0,0 & 0,2 \\
\hline Comunidad universitaria & 27,4 & 4,0 & 6,7 & 1,2 & 1,6 & 14,4 \\
\hline Externos & 1,9 & 29,3 & 0,0 & 0,0 & 46,8 & 9,4 \\
\hline $\begin{array}{l}\text { Otros usuarios (no } \\
\text { identificados como } \\
\text { universitarios) }\end{array}$ & 7,4 & 5,3 & 0,0 & 0,0 & 0,0 & 4,0 \\
\hline Universal & 50,4 & 20,0 & 46,2 & 15,1 & 0,0 & 35,5 \\
\hline PAS & 0,0 & 0,0 & 0,0 & 0,0 & 0,0 & 0,0 \\
\hline Totales & 100,0 & 100,0 & 100,0 & 100,0 & 100,0 & 100,0 \\
\hline
\end{tabular}

\section{Conclusiones}

1. El uso que las universidades españolas dan a sus perfiles oficiales en Twitter presenta rasgos uniformes, al menos por lo que a las instituciones más activas en la red se refiere. El tipo de información que suministran atiende de modo preferente a ciertos ámbitos de la actualidad universitaria, en concreto la docencia y la cultura, al tiempo que descuida áreas determinantes en la actividad de cualquier institución de educación superior; en este sentido, resulta llamativa la escasa difusión de temas relacionados con la investigación. A día de hoy, con el auge de los ránquines internacionales y la necesidad generalizada de progresar en ellos, la actividad investigadora se ha convertido en la piedra de toque de la reputación de las universidades. Creemos que su escasa presencia en Twitter sólo contribuye a que los campus españoles trasladen una imagen de marca defectuosa. Quizá el hecho de que la comunidad estudiantil -en torno a la que gravita el empleo de las social networks-participe sólo tangencialmente en las tareas de investigación, explique su limitada incidencia en esta red.

2. También se aprecian limitaciones en el tratamiento de cuestiones que trascienden la actualidad de las propias universidades. Rara vez hay en los tweets referencias a las instituciones públicas o privadas del entorno o al conjunto del sistema educativo, lo que a nuestro entender constituye un rasgo empobrecedor.

3. Por último, en relación con los públicos, hay un colectivo claramente prioritario: el de los alumnos. Frente a ellos, el PDI y el PAS no parece ser un destinatario preferente en Twitter, o no más que los usuarios externos. Ninguna universidad concibe esta red social como vía de comunicación con sus empleados; entendemos que, con ellos, el contacto se lleva a cabo por los cauces tradicionales, algo que puede responder tanto a una falta de visión estratégica por 
parte de las instituciones como a la poca adecuación del personal universitario al entorno 2.0.

\section{Bibliografía}

AGUILERA, M.; FARIAS, P. y BARAYBAR, A. (2010). "La comunicación universitaria: modelos, tendencias y herramientas para una nueva relación con sus públicos". En: Icono14, año 8, vol. 2, pp. 90-124. Disponible en: http://www.icono14. net/Num.-16.-Nuevos-tiempos-para-la-comunicacion/la-comunicacion-universitaria [27-06-2013].

ALTAMIRANO, E.; NAVA, A. y MOJICA, A. (2009). "Microblogging en la educación". En: Revista Digital Alternativa, n 6 (20), pp. 33-41.

ARANA, P. et al. (2012). "Twitter en la Universidad". En VV. AA. (2012): La universidad: una institución de la sociedad. Barcelona: Universitat Pompeu Fabra.

CELAYA, J. (2008). La empresa en la Web 2.0. Barcelona: Gestión 2000.

EBNER, M. et al. (2010). "Microblogs in Higher Education. A chance to facilitate informal and process-oriented learning?". En: Computers \& Education, $n^{\circ} 55$ (1), pp. $92-100$.

EL MUNDO (2013). "Facebook tiene 18 millones de usuarios en España y el 72\% entra con el móvil", 06-09-2013. Disponible en: http://www.elmundo.es/ elmundo/2013/09/06/navegante/1378465402.html [2-10-2013].

GÓMEZ, M.; ROSES, S. y FARIAS, P. (2011). "El uso académico de las redes sociales en universitarios". En: Comunicar, no 38, pp. 131-138.

GUZMÁN, A. et al. (2012). "Usos de Twitter en las universidades iberoamericanas". En: Revista Latinoamericana de Tecnología Educativa, $\mathrm{n}^{\circ} 11$ (1), pp. 27-39. (2013). "Impacto de Twitter en la comunicación y promoción institucional de las universidades". En: Pixel-Bit: Revista de Medios y Educación, no 43, pp. 139-153.

IGARTUA, J. J. (2006). Métodos cuantitativos de investigación en comunicación. Barcelona: Bosch.

KAPLAN, A. y HAENLEIN, M. (2010). "Users of the world, unite! The challenges and opportunities of social media". En: Business Horizon, vol. 53, $\mathrm{n}^{\circ}$ 1, pp. 59-68.

KIERKEGAARD, S. (2010). "Twitter thou doeth?". En: Computer Law \& Security Review, $\mathrm{n}^{\circ} 26$ (6), pp. 577-594.

LA VANGUARDIA (2013). "Twitter suma más usuarios y facturación, pero también pérdidas", 16-10-2013. Disponible en: http:/www.lavanguardia.com/economia/20131016/54392061931/twitter-mas-usuarios-facturacion-y-perdidas.html [24-10-2013].

PANIAGUA, F. J.; GÓMEZ CALDERÓN, B. y FERNÁNDEZ, M. (2012). "La incorporación de los departamentos de comunicación de las universidades españolas al entorno digital. Un análisis cuantitativo". En: Estudios sobre el Mensaje Periodístico, $\mathrm{n}^{\mathrm{o}} 18$ (3), pp. 691-701.

REGALADO, O. (2011). ¿Cómo deberían usar las universidades las redes sociales?”. Disponible en: http://www.dosensocial.com/category/dosensocial [02-06-2013]. 
REINA, J.; FERNÁNDEZ, I. y NOGUER, Á. (2012). "El uso de las redes sociales en las universidades andaluzas: El caso de Facebook y Twitter". En: Revista Internacional de Relaciones Públicas, $\mathrm{n}^{\circ}$ 4, vol. II, pp. 123-144.

RINALDO, S.; TAPP, S. y LAVERIE, D. (2011). "Learning by tweeting”. En: Journal of Marketing Education, $\mathrm{n}^{\circ} 33$ (2), pp. 193-203.

SÁNCHEZ, J. J. (2005). "Análisis de contenido cuantitativo de medios". En BERGANZA, M. R. y RUIZ, J. A. (2005). Investigar en Comunicación. Madrid: McGraw Hill, pp. 207-228.

TAPIA, A. et al. (2010). "Los estudiantes universitarios ante las redes sociales: cuestiones de uso y agrupación en estructuras elitistas o pluralistas". En: Revista Vivat Academia, $\mathrm{n}^{\mathrm{o}}$ 113. Disponible en: http://www.ucm.es/info/vivataca/numeros/ n113/DATOSS.htm [23-06-2013].

VELETSIANOS, G. (2012). "Higher education scholars' participation and practices on Twitter". En: Journal of Computer Assisted Learning, nº 28 (4), pp. 336-349.

\section{Notas}

1 Entendemos aquí por redes sociales lo que Kaplan y Haenlein (2010: 61) definen como "a group of Internet-based applications that build on the ideological and technological foundations of Web 2.0, and that allow the creation and exchange of Used Generated Contents".

2 Cifra ofrecida por la compañía en el informe de gestión correspondiente al tercer trimestre de 2013 (La Vanguardia, 2013).

3 Se refiere a usuarios activos al mes, de los cuales el 61 por ciento consulta la red a diario (El Mundo, 2013).

4 Hay sólo dos universidades que carecen de perfil institucional en Twitter, las de Lleida y Pontificia de Salamanca, si bien la primera dispone de cuentas referidas a parcelas concretas de su actividad, como investigación o emprendimiento.

5 Esto contradice las conclusiones de estudios aplicados a instituciones de educación superior de otros ámbitos geográficos, como el de Guzmán et al. (2013: 147), que asegura que "las Universidades con más estudiantes tienen más seguidores y publican más tweets". No es así en el caso español.

6 De acuerdo con este trabajo, los temas más frecuentes de los tweets, ordenados por número de incidencias, son: divulgación de campañas de promoción de servicios, difusión de la programación cultural, interacción entre alumnos y profesores, comunicación de investigaciones y proyectos, promoción de los servicios de biblioteca, convocatoria para participar en actividades de interés social e invitación a seminarios y congresos.

7 Es decir, aquel que se presenta como perfil institucional de la universidad. Muchas disponen, además, de cuentas especializadas mantenidas por servicios específicos (cultura, investigación, biblioteca...), que no se han contemplado aquí.

8 El perfil oficial de la Universidad de Santiago de Compostela (@visitasUSC) presenta un promedio de 10,55 tweets al día pero, dado que no es de carácter generalista sino que se centra en la difusión del patrimonio cultural y artístico de la institución, se decidió excluirlo. En el caso de universidades con cuentas institucionales en varias lenguas (Oberta de Catalunya, Barcelona y Jaume I), se operó con el total agregado de mensajes. 


\section{Los autores}

Bernardo José Gómez Calderón. Profesor de Periodismo de la Universidad de Málaga. Ha publicado, entre otras obras, Ladrón de fuego. La obra en prensa de Francisco Umbral (2004) y, en colaboración con Teodoro León Gross, El artículo literario: Manuel Alcántara (2008) y Diez articulistas para la historia de la literatura española (2009), además de una veintena de artículos en revistas académicas de impacto. Suma dieciséis capítulos de libros, y ha asistido como comunicador y ponente a medio centenar de congresos nacionales e internacionales. Coordinó entre 2005 y 2011 el Informe anual de la profesión periodística editado por la Asociación de la Prensa de Madrid.

Francisco Javier Paniagua Rojano. Profesor de Periodismo de la Universidad de Málaga. Desde 2005 a 2011 coordinó el Informe anual de la Profesión Periodística editado por la Asociación de la Prensa de Madrid y en 2012 participó como coautor del capítulo "Journalist in Spain" en el estudio Global Journalist in the 21st Century, dirigido por David Weaver y Lars Willnat. Desde 2008 es profesor del máster en Comunicación Política e Institucional de la Fundación Ortega y Gasset. Ha publicado varios artículos en revistas como Estudio sobre el Mensaje Periodístico, Latina de Comunicación Social, Icono 14 o Ámbitos, sobre comunicación institucional y profesión periodística. Es secretario de la sección 7 (Comunicación estratégica y organizacional) de la Asociación Española de Investigadores de la Comunicación (AE-IC). 